\title{
Neurodegenerative Diseases Stem Cell-based Therapeutic: A Perspective
}

\section{Kuldip S Sidhu}

Centre for Healthy Brain Ageing, UNSW Medicine, UNSW, Sydney Australia

Corresponding author: A/Prof. Kuldip S Sidhu, Centre for Healthy Brain Ageing, UNSW Medicine, UNSW, Sydney, NSW 2152, Australia, Tel: +61401766055; E-mail: k.sidhu@unsw.edu.au

Received: Feb 20, 2016; Accepted: Feb 26, 2016; Published: Feb 29, 2016

\section{Opinion}

The term neurodegeneration encompasses a broad range of diseases of central and peripheral nervous system. It's only in less than $5 \%$ of the cases a clear genetic link has been established, however, majority is sporadic and driven by a combination of genetic and environmental factors. Alzheimer's and Parkinson's are the most common and renowned disorders that largely affect the elderly population. However, neural degeneration and associated loss of memory in these diseases is not a normal part of ageing as it occurs only in few, not all elderly people. It is considered that neurodegeneration is a generalized systemic disorder that also inflicts other organs, although it is most pervasive in neurons. Interestingly, it is not cell-autonomous, that means multiple cell types such as astrocytes, oligodendroglia, Schwann cells etc. are also involved. Thus investigating the cause of neurodegeneration at the cellular and sub-cellular levels is most challenging. Since initially only few cells are involved, it is impossible to access those cells in human and animal models. The post-mortem tissue although relevant but offer only retrospective indicator of disease. The current modalities are only symptomatic treatment with no cure in horizon. Therefore, therapeutic interventions for neurodegenerative diseases remain the most daunting task today. The emerging new technology like stem cells based therapeutics can offer some respite and is becoming an active area of research.

There has been an unparallel development during the last 2-3 decades in small molecules and biologics such as insulin, growth factors, antibodies, promoters and inhibitors of various metabolic pathways to modify the disease process and these are known as the 1st and 2 nd pillar of human therapeutics. Currently stem cells including other types of therapeutic cells are sitting on the cusp of another revolution, emerging as the 3rd pillar of human medicine. However, the complexities of living cells and their interactions with host tissue pose a significant new challenge not encountered before in human medicine. But nevertheless stem cells can offer cure rather treatment for various ailments particularly those involving loss or degeneration of specific cells like in Diabetes, Spinal Cord Injury, Alzheimer's and Parkinson's diseases. There are emerging new standards for mimicking true human physiology like engineering organ on chip like mini brain in the Petri dish so that responses to medicine, chemicals and diseases can be accurately predicted in human cells/organoids as supposed to that in animals. The attempts are to support and repair damaged tissues and organs by providing healthy stem cell- derived transplants. Bone marrow stem cell transplants for cancer patients have been successfully used for over 20 years now.

Why stem cells, because they all depict three basic characteristic features that make them special for their use in therapeutics such as proliferation (divide rapidly), self-renewal (immortal) and differentiation to different cell types (multiand/or pluripotent). There has been a significant progress to harness these potentials of stem cells so that they could be used potentially and effectively in human therapeutics. Generally there are two broad categories of stem cells, pluripotent stem cells (embryonic and induced pluripotent stem cells) that are derived from embryos or by changing fate of the somatic cells and adult stem cells that are derived from adult tissues and are by and large multi-potent. Both types of stem cells are progressing towards human therapeutics but adult stem cells have an edge over embryonic stem cells because of ethical and safety concerns with the latter. Another added advantage of adult stem cells is that since these can be derived from the same patient thus once transplanted back are less likely to be rejected because they will be autologous. The pluripotent stem cells will remain the analogous source of cells and will be likely to be rejected if transplanted. But since they are pluripotent, virtually every single cell in our body can generated from them as supposed to in adult stem cells where they have restricted differentiation profiles. In literature a balance approach is being used for both kinds of stem cells in developing human therapeutics.

These stem cells have potential to cure rather treat human diseases particularly those that are characterized by loss of single cell type in the body as mentioned earlier. According to the recent World Health Organization's large-scale studies, about a third of the adult worldwide population suffer from a mental disorder such as depression, anxiety and schizophrenia. If also taken together with neurological disorders, such as dementia and stroke, these "disorders of the brain" account for $13 \%$ of the global disease burden. This surpasses both cardiovascular diseases (5\%) and cancer (10\%). Countries with the highest rate of burden $>650$ Disability Adjusted Life Years [DALYs] per 100,000 population included the USA, UK, Russia, and Australia [1].

There are emerging data indicating that now stem cells based therapeutics may be in the horizon soon. However, translating these ideas to clinic is a cliff hanger at the moment. There are number of issues. There seems to be a significant gap between public expectations and the scientific reality to 
accomplish clinical translation. But nevertheless there are unprecedented developments seen recently in this field with proof of principle emerging for number of diseases that currently have no cure but only management, like Alzheimer's, Parkinson's spinal cord injury, to name a few where stem cellbased therapies (regenerative medicine) can offer respite [2]. Despite huge progress and potentials, there remain scientific, technical, logistic, financial and administrative/regulatory obligations that are required to be met to move this field forward.

The regenerative medicine being a new concept there is no single harmonized recommendation that can be used to describe the preclinical guidelines or model for this therapeutics. Safety, efficacy, cell viability and tracing such cells are the inherent challenges in stem cell-based therapeutics. The existing model for clinical trials with stem cell products is not clear or explicit and this possesses a significant roadblock for transition to clinics. Perhaps the route followed by the blood and bone marrow transplantation initiatives may offer respite i.e., the need-based control human trials and case studies. However, sprouting of spurious stem cells clinics worldwide and the associated patient tourism is a big concern that needs addressing perhaps by proper educational initiatives in this area. Medical tourism is nothing new and there is nothing wrong but mushrooming of spurious stem cells clinics' offering false hope to vulnerable patients is a concern. Majority of these of the shelf clinics have emerged around cosmetic surgery without legitimate quality control and that remains the major concern of the regulatory agencies.
The clinical translation from lab to bedside using stem cells is a cliff hanger and still requires liberal investments. Each drug discovery costs around 1.4 billion US $\$$ and it is likely to be more for stem cells-based therapy and that thus cannot be supported with public funding. Thus the existing drug delivery model won't work, and an alternative viable model is required. A need based case control studies may be encouraged as was done for developing bone marrow stem cells transplantation. As is always the new area like this involves risks that are hampering private investments in this field. However, similar skepticism for investments was there for DNA recombinant technology and human genome projects; both are now providing dividend far excess than investments made. High risk venture capital investments are required. In the absence of significant venture capital and major pharmaceutical company interest, an alternative perspective is needed for academic stem cell scientists to partner with industry biotechnology expertise thus add value to quality controls in such settings and to build confidence with regulatory agencies.

\section{References}

1. Sidhu KS, Kateb B (2014) World Brain Mapping and Therapeutic Initiative: A proposed G20 priority due to major impact of the cost of neurological disorders on the world economy. J Neurol Disord 2: 1-2.

2. Avior Y, Sagi I, Benvenisty N (2016) Pluripotent stem cells in disease modelling and drug discovery. Nat Rev Mol Cell Biol 17: 170-182. 